UND-HEP-97-BIG 06

TTP97-33

hep-ph/9708372

\title{
Non-Abelian Dipole Radiation and the Heavy Quark Expansion
}

\author{
A. Czarnecki ${ }^{a}$, K. Melnikov ${ }^{a}$ and N. Uraltsev ${ }^{b, c}$ \\ ${ }^{a}$ Institut für Theoretische Teilchenphysik, \\ Universität Karlsruhe, D-76128 Karlsruhe, Germany \\ ${ }^{b}$ Dept. of Physics, Univ. of Notre Dame du Lac, Notre Dame, IN 46556, U.S.A. \\ ${ }^{c}$ St. Petersburg Nuclear Physics Institute, Gatchina, St. Petersburg 188350, Russid
}

\begin{abstract}
Dipole radiation in QCD is derived to the second order in $\alpha_{s}$. A power-like evolution of the spin-singlet heavy quark operators is obtained to the same accuracy. In particular, $\mathcal{O}\left(\alpha_{s}^{2}\right)$ relation between a short-distance low-scale running heavy quark mass and the $\overline{\mathrm{MS}}$ mass is given. We discuss the properties of the effective QCD coupling $\alpha_{s}^{(\omega)}(E)$ which governs the dipole radiation. This coupling is advantageous for heavy quark physics.
\end{abstract}

${ }^{*}$ Permanent address 
Theoretical description of heavy flavor decays benefits from a strong hierarchy between the mass of the decaying quark and the typical scale of the strong interactions, $m_{b(c)} \gg \Lambda_{\mathrm{QCD}}$. A current level of experimental precision requires an accurate treatment of nonperturbative effects even in beauty decays. A consistent genuinely QCD-based framework for such a treatment is provided by the heavy quark expansion (HQE). The HQE combines the Wilson operator product expansion (OPE) separating the physics of low and high momentum scales with the nonrelativistic expansion, to treat the nonperturbative effects originating at large distances. It allows a simultaneous precision account for both perturbative and nonperturbative effects.

An important class of applications of the HQE are semileptonic weak transitions between $b$ and $c$ quarks, i.e. the situation when both initial and final quarks are heavy. In particular, the most informative predictions can be made for the transition amplitudes in the so-called small velocity (SV) limit [1], when the velocities of heavy hadrons in initial and final state are small. The heavy quark in this case plays a role of a static (or slowly moving) source of the color Coulomb field which affects the light degrees of freedom in the hadrons. This physical picture is formalized in the Wilsonian field-theory approach by integrating out high-momentum degrees of freedom of full QCD and resorting to an effective low-energy theory, where highly excited hadronic states are not present.

A peculiarity of the effective theory for heavy flavor hadrons is its essentially Minkowskian nature. In QCD any process in which the velocity of the heavy quark $Q$ changes, involves actual gluon radiation with energy and momentum in the whole range up to the quark masses. On the other hand, effective theories are called upon to eliminate all high-momentum physics. Such a problem does not arise in usual effective theories (typically formulated in the Euclidean space) where all short-distance physics is virtual. This necessitates a careful control over the radiation effects.

On the other hand, when the energy loss is small compared to $m_{Q}$, the radiation off the heavy colored particles is almost a classical effect, and therefore a universal process-independent description is possible. In the limit of small velocity it is a familiar dipole radiation. It has, of course, some peculiarities in the non-Abelian theories like QCD.

Even though the dipole radiation has the most obvious manifestation in the SV processes, it is relevant in a more general context, for example, for zero-recoil processes or inclusive decay widths. The reason is that the OPE ensures that the effects of soft physics originating at the momentum scale well below $m_{Q}$ enter all genuinely short-distance observables in a universal way, via its contribution to the local heavyquark operators. The knowledge of the perturbative dipole radiation allows one to determine the short-distance evolution of a number of composite operators in the effective theory of heavy quarks.

In the present paper, we derive the non-Abelian dipole radiation to the second order in $\alpha_{s}$ and use this result to obtain a power evolution of a number of spinsinglet heavy quark operators to order $\alpha_{s}^{2}$. In particular, a gauge-invariant relation between a short-distance low-scale running heavy quark mass and the $\overline{\mathrm{MS}}$ mass 
$\bar{m}_{Q}\left(m_{Q}\right)$ is given, which becomes a practical necessity since the two-loop accuracy in the processes with heavy quarks is becoming the state of the art.

The radiation by the heavy charged particle occurring when its velocity changes is a well-known effect from classical electrodynamics. It is most simply obtained by a direct computation of the Lienard-Wiechert retarded potentials $A_{\mu}(\vec{r}, t)$ at $\vec{r} \rightarrow \infty$, and has the form

$$
\frac{1}{\omega} \frac{\mathrm{d} I(\omega)}{\mathrm{d} \omega}=\frac{\alpha}{\pi}\left(\frac{1}{|\vec{v}|} \ln \frac{1+|\vec{v}|}{1-|\vec{v}|}-2\right) \frac{1}{\omega}=\frac{2}{3} \frac{\alpha}{\pi} \frac{\vec{v}^{2}}{\omega}+\mathcal{O}\left(\vec{v}^{4}\right),
$$

where $\omega$ is the radiated energy, $I(\omega)$ is intensity and $\alpha$ is the fine structure constant. We will be interested only in the dipole term $\propto \vec{v}^{2}$ in QCD, and do not consider multipole radiation proportional to higher powers of $\vec{v}$.

In quantum electrodynamics the same relation holds, with $1 / \omega \mathrm{d} I / \mathrm{d} \omega$ giving the probability to radiate soft photon(s) with energy $\omega$. Moreover, there are no higherorder corrections in $\alpha_{\mathrm{em}}$, provided $\omega \ll m$ and the recoil effects of the radiating particle $m$ are neglected. Nontrivial corrections are suppressed by powers of $\omega / \mathrm{m}$.

A certain type of corrections emerge only due to effects of polarization of the quantum vacuum by dipole radiation. It is, therefore, directly related to the running of the gauge coupling in the quantum field theory. The radiation of the real (on-shell) photons is proportional to the physical value of the fine structure constant $\alpha \equiv \alpha_{\mathrm{em}}(0)$. However, because of the pair production, the radiated energy is governed by the running coupling $\alpha(\omega)$. For small $\omega$, all vacuum polarization effects are suppressed by powers of $\omega^{2} / m_{e}^{2}$ where $m_{e}$ is the lightest charged particle. Therefore, the soft radiation in QED is defined by $\alpha(0)$ without any correction, in accord with classical electrodynamics. This is expected since semiclassical approximation is parametrically justified in the limit $\omega \rightarrow 0$.

The dipole radiation in the non-Abelian theory is quite different. Because of gluon self-interaction, the effective coupling increases even in the absence of light flavors (it also becomes different for different multipoles). Eventually it grows up to values of order 1 and the theory enters the strong interaction phase. At such energies nonperturbative effects emerge which generate color confinement shaping the spectrum of observed hadrons.

Our main interest lies in the domain where $\omega$ is large compared to $\Lambda_{\mathrm{QCD}}$ (the nonperturbative multipole expansion of the color-singlet systems was discussed in [2]). In this regime, perturbative calculations can be performed. Below we introduce a few standard notations used for heavy quarks.

Let us consider a general process of scattering of an external color-singlet weak current $J$ with momentum $q$ on a heavy quark $Q$ in the SV kinematics. For simplicity, the initial quark is assumed to be at rest. The initial $Q$ and final state $\tilde{Q}$ quarks can have arbitrary masses; however, both masses must be large, so that the nonrelativistic expansion can be applied. The SV limit $\vec{v}=\vec{q} / m_{\tilde{Q}}, \quad|\vec{v}| \ll 1$ is kept by adjusting $\vec{q}$ appropriately. 
The current $J$ must have a non-vanishing tree level nonrelativistic limit, otherwise it can be arbitrary. The most familiar cases are the time-like component of the vector current $J_{0}^{\dagger}=\bar{Q} \gamma_{0} \tilde{Q}$ or the scalar current $J_{S}^{\dagger}=\bar{Q} \tilde{Q}$.

The inclusive processes of the scattering on heavy quarks are described by the corresponding structure function $w\left(q_{0}, \vec{q}\right)$ which is a sum of all transition probabilities induced by $J$ into the final states with momentum $\vec{q}$ and energy $m_{Q}+q_{0}$. Using the optical theorem, one represents the structure function $w$ as a discontinuity of the forward transition amplitude $T\left(q_{0}, \vec{q}\right)$ at physical values of $q_{0}$ :

$$
T\left(q_{0}, \vec{q}\right)=\frac{i}{2 m_{Q}} \int d^{4} x \mathrm{e}^{-i q x}\left\langle Q\left|T J(x) J^{\dagger}(0)\right| Q\right\rangle, w\left(q_{0}, \vec{q}\right)=2 \operatorname{Im} T\left(q_{0}, \vec{q}\right) .
$$

The causality ensures that $T\left(q_{0}, \vec{q}\right)$ is an analytic function of $q_{0}$ in the upper halfplane. In the heavy quark limit the spin degrees of freedom become irrelevant, and Eqs. (2) assume averaging over spin states (also color, for perturbative calculations, etc.).

The structure functions have a threshold $q_{0}^{\min }$ corresponding to the elastic transition:

$$
q_{0}^{\min }=\sqrt{\vec{q}^{2}+m_{\tilde{Q}}^{2}}-m_{Q} \simeq m_{\tilde{Q}}-m_{Q}+\frac{m_{\tilde{Q}} \vec{v}^{2}}{2} .
$$

The variable $\omega$ measures the hadron excitation energy in the final state:

$$
\omega=q_{0}-q_{0}^{\min } \simeq q_{0}+m_{Q}-m_{\tilde{Q}}-\frac{m_{\tilde{Q}} \vec{v}^{2}}{2}
$$

For simplicity, we consider below the case of equal masses, $m_{\tilde{Q}}=m_{Q}$, although nothing depends on this assumption.

The nonrelativistic expansion implies that $\omega \ll m_{Q}$. On the other hand, the perturbative treatment is justified at $\omega \gg \Lambda_{\mathrm{QCD}}$, and this hierarchy will be assumed in what follows. The nonperturbative aspects will be addressed afterwards. Resorting to the perturbative calculations, we can use the quark states instead of actual heavy hadrons, which was tacitly assumed above.

The structure function $w$ takes the following form in the heavy quark limit:

$$
w(\omega, \vec{v})=N \delta(\omega)+\frac{2 \vec{v}^{2}}{3} \frac{d(\omega)}{\omega}+\mathcal{O}\left(\vec{v}^{4}\right)
$$

At $\vec{v}=0$ only the elastic peak is present. The excitations, described by the second term, appear at the level $\sim 1 / m_{Q}^{2}$ or $\vec{v}^{2}$ [1]. The dipole radiation is described by the function $d(\omega)$.

Motivated by the dipole radiation in QED, we define a coupling $\alpha_{s}^{(\omega)}$ by projecting Eq. (5) on its second term:

$$
C_{F} \frac{\alpha_{s}^{(\omega)}(\omega)}{\pi \omega}=\lim _{\vec{v} \rightarrow 0} \lim _{m_{Q} \rightarrow \infty} \frac{3}{2 \vec{v}^{2}} \frac{w(\omega, \vec{v})}{\int_{0}^{\omega} w\left(\omega^{\prime}, \vec{v}\right) \mathrm{d} \omega^{\prime}} .
$$


The denominator in the last ratio is necessary to get rid of the overall normalization of the effective nonrelativistic current (renormalized down to the momentum scale near $\omega)$. In this form the heavy quark limit $m_{Q} \rightarrow \infty$ yields a finite result. It is important that the normalization integral includes also the elastic peak, which makes it infrared safe at arbitrary $\vec{v}$. On the other hand, the exact upper limit does not matter in the SV kinematics, since it affects the ratio only by corrections $\sim \vec{v}^{2}$.

Therefore, the inelastic structure function in the SV limit determines the effective QCD running coupling driving the dipole radiation of gluons. It is a dimensionless quantity, and thus is a function of the ratio $\Lambda_{\mathrm{QCD}} / \omega$. The explicit coefficient in Eq. (6) is adjusted to make $\alpha_{s}^{(\omega)}$ equal to the bare coupling in the Born approximation.

It is important that the OPE and factorization of the infrared effects $\square$ ensures that $\alpha_{s}^{(\omega)}(\omega)$ is universal, in the sense that:

a) it does not depend on the choice of the weak current $J$ probing the heavy quark structure, or on the ratio of the quark masses;

b) as long as the onset of the quark-hadron duality is passed, there is no dependence on the particular type of the initial heavy hadron. This property always holds in the perturbative analysis.

The effective coupling $\alpha_{s}^{(\omega)}$ obeys the usual renormalization group evolution

$$
\begin{gathered}
\omega \frac{\mathrm{d}}{\mathrm{d} \omega} \frac{\alpha_{s}^{(\omega)}(\omega)}{\pi}=-\beta\left(\frac{\alpha_{s}^{(\omega)}(\omega)}{\pi}\right)=-\frac{\beta_{0}}{2}\left(\frac{\alpha_{s}^{(\omega)}(\omega)}{\pi}\right)^{2}-\frac{\beta_{1}}{8}\left(\frac{\alpha_{s}^{(\omega)}(\omega)}{\pi}\right)^{3}-\ldots, \\
\beta_{0}=\frac{11}{3} C_{A}-\frac{2}{3} n_{f}, \quad \beta_{1}=102-\frac{38}{3} n_{f} .
\end{gathered}
$$

The coefficients $\beta_{2}$ and higher are scheme-dependent and therefore differ from the standard ones obtained for the $\overline{\mathrm{MS}}$ coupling. Note that because of its physical definition, the evolution of $\alpha_{s}^{(\omega)}$ is properly defined when heavy flavor thresholds are passed.

Although the coupling $\alpha_{s}^{(\omega)}$ is most natural for heavy quark decays, in practice one needs to know its relation to the standard reference coupling $\alpha_{s}^{\overline{\mathrm{MS}}}$. We calculated it to the second order in $\alpha_{s}^{2}$ using the technique of the Ref. [3]:

$$
\begin{aligned}
\frac{\alpha_{s}^{(\omega)}(\mu)}{\pi}= & \frac{\alpha_{s}^{\overline{\mathrm{MS}}}(\mu)}{\pi}+\left[\left(\frac{5}{3}-\ln 2\right) \frac{\beta_{0}}{2}-C_{A}\left(\frac{\pi^{2}}{6}-\frac{13}{12}\right)\right]\left(\frac{\alpha_{s}}{\pi}\right)^{2}+\mathcal{O}\left(\alpha_{s}^{3}\right)= \\
& =\frac{\alpha_{s}^{\overline{\mathrm{MS}}}\left(\mathrm{e}^{-5 / 3+\ln 2} \mu\right)}{\pi}-C_{A}\left(\frac{\pi^{2}}{6}-\frac{13}{12}\right)\left(\frac{\alpha_{s}}{\pi}\right)^{2}+\mathcal{O}\left(\alpha_{s}^{3}\right),
\end{aligned}
$$

where $C_{A}=N_{c}$ for the $\mathrm{SU}\left(N_{c}\right)$ gauge group.

In the latter form we absorbed the effect of running $\alpha_{s}$ into the redefinition of the scale at which the $\overline{\mathrm{MS}}$ coupling is evaluated 四. The term $\sim C_{A}$ represents the so-called genuine (non-BLM) second-order effect; it has purely non-Abelian origin.

\footnotetext{
${ }^{1}$ Physically, it is merely existence of the effective low-energy theory of heavy quarks.
} 
We find that its coefficient is not particularly large, -1.685; it is not small either as it happens in a number of other observables. Let us note that a similar effective

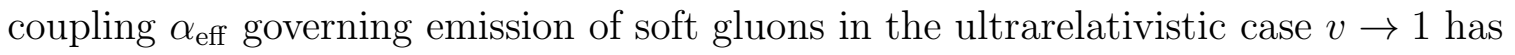
been also calculated to two loops [5]:

$$
\frac{\alpha_{\mathrm{eff}}(\mu)}{\pi}=\frac{\alpha_{s}^{\overline{\mathrm{MS}}}(\mu)}{\pi}+\left[\frac{5}{6} \frac{\beta_{0}}{2}-C_{A}\left(\frac{\pi^{2}}{12}-\frac{1}{3}\right)\right]\left(\frac{\alpha_{s}}{\pi}\right)^{2}
$$

its exact definition, however, is less direct and more complicated. It differs from $\alpha_{s}^{(\omega)}$ in the second-order terms, although the difference is small numerically.

The BLM-type terms which account for the effects of running of the QCD coupling in the leading-order calculations can be easily computed to any order in this case.2] They contain corrections $\sim\left(\beta_{0} \alpha_{s} / \pi\right)^{k}$ where the factor $\beta_{0}$ is singled out by the dependence on the number of light flavors [7]. For example, the BLM estimate of the $\alpha_{s}^{3}$ term in the last Eq. (8) is

$$
\mathcal{O}\left(\alpha_{s}^{3}\right) \stackrel{\mathrm{BLM}}{=}-\left(\frac{\pi^{2}}{6}-\frac{31}{36}\right)\left(\frac{\beta_{0}}{2}\right)^{2}\left(\frac{\alpha_{s}}{\pi}\right)^{3}, \quad \frac{\pi^{2}}{6}-\frac{31}{36} \simeq 0.78 .
$$

For book-keeping purposes we also give here the massive quark $\mathcal{O}\left(\alpha_{s}\right)^{2}$ contribution to $\alpha_{s}^{(\omega)}$ : with an additional massive quark $q$ the extra term in the last Eq. (8) is given by

$-\frac{1}{6}\left(\frac{\alpha_{s}}{\pi}\right)^{2}\left\{\ln x-4 \ln 2+\frac{10}{3}+\vartheta(1-x) \int_{x}^{1} \frac{d t}{t}\left(1+\frac{t}{2}\right) \sqrt{1-t}\left(1+\frac{x}{2 t}\right) \sqrt{1-\frac{x}{t}}\right\}$,

where $x=4 m_{q}^{2} / \mu^{2}$ and $\alpha_{s}^{\overline{\mathrm{MS}}}(\mu)$ is assumed to be defined for $n_{f}+1$ light flavor.

A construction of the HQE in QCD requires an accurate definition of the basic objects of the effective theory, in the first place the heavy quark mass, kinetic operator and all other composite operators. Being defined consistently, all they depend on normalization point. A normalization-point-independent pole mass of the heavy quark, although appearing in the purely perturbative calculations at a given order, cannot be completely defined at the level when the nonperturbative effects are addressed. By the same token, the parameter $\bar{\Lambda}$ measuring the difference between the heavy hadron mass and $m_{Q}$ in the heavy quark limit, suffers from a similar uncertainty. Instead, one must use the short-distance mass $m_{Q}(\mu)$ and, correspondingly, $\bar{\Lambda}(\mu)=\lim _{m_{Q} \rightarrow \infty} M_{H_{Q}}-m_{Q}(\mu)$ with $\Lambda_{\mathrm{QCD}} \ll \mu \ll m_{Q}$ [8]. The low-scale short distance mass can be defined in different ways. We recall, however, that the standard $\overline{\mathrm{MS}}$ mass cannot be used for $\mu \ll m_{Q}$ [9]. Problems, similar to those in the pole mass, also emerge in attempts to define spin-singlet operators without a powerlike mixing with lower-dimension operators, including the unit operator.

\footnotetext{
${ }^{2}$ All necessary expressions are given in $[6] ; c_{\pi}$ in Eq. (28) is just $C_{F} \cdot \alpha_{s}^{(\omega)}(\mu) / \pi$ in the BLM approximation. The BLM series has a finite radius of convergence, $4 / \beta_{0}\left(1+\frac{25}{9 \pi^{2}}\right)^{-1 / 2}$ in terms of $\alpha_{s}^{\overline{\mathrm{MS}}}(\mu)$, or $4 / \beta_{0}$ in terms of $\alpha_{s}^{\overline{\mathrm{MS}}}\left(\mu \mathrm{e}^{-5 / 6}\right)$.
} 
The physically appropriate gauge-invariant scheme was suggested in [6, 10, 9] and is based on the SV sum rules relating the moments of the SV structure functions to the local heavy quark operators. The normalization point is introduced as an upper cutoff in the integral over excitation energy. For example, for a heavy hadron $H_{Q}$ one defines

$$
\begin{gathered}
\bar{\Lambda}(\mu) \equiv \lim _{m_{Q} \rightarrow \infty}\left[M_{H_{Q}}-m_{Q}(\mu)\right]=\lim _{\vec{v} \rightarrow 0} \lim _{m_{Q} \rightarrow \infty} \frac{2}{\vec{v}^{2}} \frac{\int_{0}^{\mu} \omega w(\omega, \vec{v}) \mathrm{d} \omega}{\int_{0}^{\mu} w(\omega, \vec{v}) \mathrm{d} \omega}, \\
\mu_{\pi}^{2}(\mu) \equiv \frac{\left\langle H_{Q}\left|\bar{Q}(i \vec{D})^{2} Q\right| H_{Q}\right\rangle_{\mu}}{\left\langle H_{Q}|\bar{Q} Q| H_{Q}\right\rangle_{\mu}}=\lim _{\vec{v} \rightarrow 0} \lim _{m_{Q} \rightarrow \infty} \frac{3}{\vec{v}^{2}} \frac{\int_{0}^{\mu} \omega^{2} w(\omega, \vec{v}) \mathrm{d} \omega}{\int_{0}^{\mu} w(\omega, \vec{v}) \mathrm{d} \omega},
\end{gathered}
$$

etc. The above operator relations for the SV moments of the structure function suggest that the perturbative evolution must be driven by one and the same function. With the above definitions it is directly $\alpha_{s}^{(\omega)}(\mu)$. From Eqs. $(\sqrt[12]{12}, 13)$ one finds:

$$
\frac{\mathrm{d} \bar{\Lambda}(\mu)}{\mathrm{d} \mu}=\frac{4}{3} C_{F} \frac{\alpha_{s}^{(\omega)}(\mu)}{\pi}, \quad \frac{\mathrm{d} \mu_{\pi}^{2}(\mu)}{\mathrm{d} \mu^{2}}=C_{F} \frac{\alpha_{s}^{(\omega)}(\mu)}{\pi}
$$

where $\alpha_{s}^{(\omega)}(\mu)$ is given in Eq. (8).

We note that the right-hand side of Eq. (14) for the kinetic operator was obtained, with the $\alpha_{s}^{2}$ accuracy, in [11] considering the zero-recoil processes, where the dipole radiation is absent and the inelastic structure functions appear only in the order $1 / m_{Q}^{2}$. Once again, the OPE ensures that the result is process-independent, which is demonstrated here at the quite nontrivial level of genuine two-loop corrections. [ The SV limit, however, is technically important since the separation between high- and low-energy hadronic states by means of the cutoff in $\omega$ is velocity-independent only through order $\vec{v}^{2}$.

The similar $\mu$-dependence holds for the renormalized slope of the Isgur-Wise function when it is defined in the same physical way [9], based on the Bjorken sum rule:

$$
\mu \frac{\mathrm{d}\left[\varrho^{2}(\mu)-\frac{1}{4}\right]}{\mathrm{d} \mu}=\frac{4}{3} \frac{2 \alpha_{s}^{(\omega)}(\mu)}{3 \pi} .
$$

Due to the short-distance effects, the observable transition form factors do not have a literal heavy quark limit at $\vec{v} \neq 0$ but vanish, and require factoring out the perturbative suppression due to gluon radiation, to yield the effective $\mu$-dependent IW function $\xi\left(v^{2} ; \mu\right)$. The renormalization factors generally differ depending on the way the $\mu$-cutoff is introduced in the perturbative factors. In analogy with Eq. (11), it is convenient to define $\xi\left(v^{2} ; \mu\right)$ for small $v$ as

$$
\xi\left(v^{2} ; \mu\right)=\lim _{m_{Q} \rightarrow \infty} \frac{F(v)}{\left[\frac{1}{2 \pi} \int_{0}^{\mu} w(\omega, \vec{v}) \mathrm{d} \omega\right]^{1 / 2}}, \quad \varrho^{2}(\mu)=-\left.2 \frac{\mathrm{d} \xi\left(v^{2} ; \mu\right)}{\mathrm{d} \vec{v}^{2}}\right|_{v=0},
$$

\footnotetext{
${ }^{3}$ To first order in $\alpha_{s}$ it was checked in [13], and, for the BLM terms, in [6] to all orders.
} 
where $F$ is the form factor, for example, of the vector current $\bar{Q} \gamma_{0} Q$ and $w$ is the corresponding structure function. This is the scheme suggested in [9] for which Eq. (15) holds. Various definitions using the dimensional subtraction schemes differ from this one; they do not have such a direct physical meaning, however. It is interesting to note that the perturbative effects in $\varrho^{2}(\mu)$ can be viewed as a universal $\mu$-dependent renormalization of the tree-level constant accompanying it $(-1 / 4$ for scalar mesons or 0 for baryons).

The RG equations (14) are directly proportional to $\alpha_{s}^{(\omega)}$. Hence, the application of the OPE shows that the first-order running is not renormalized in the Abelian theory (without light fermions) to all orders in the coupling [6], since in such a theory $\alpha_{s}^{(\omega)}$ identically coincides with $\alpha(0)$, as discussed above.

According to [10], one can calculate perturbatively a physical (gauge-invariant) low-scale running mass $m_{Q}(\mu)$ with $\mu \ll m_{Q}$ by subtracting, order by order, the infrared part given by the SV sum rules, from the pole mass $m_{Q}^{\text {pole }}$ :

$$
m_{Q}(\mu)=\left[m_{Q}^{\text {pole }}\right]_{\text {pert }}-\left[\Lambda_{\mathrm{QCD}}(\mu)\right]_{\text {pert }}-\frac{1}{2 m_{Q}(\mu)}\left[\mu_{\pi}^{2}(\mu)\right]_{\text {pert }} .
$$

This mass determines the kinetic energy term in the renormalized heavy quark Hamiltonian when it is expanded in the velocity of the heavy quark:

$$
\mathcal{H}_{Q}=\bar{Q} A_{0} Q+\frac{1}{2 m_{Q}(\mu)} \bar{Q}\left((i \vec{D})^{2}-c_{G} \frac{i}{2} \sigma G\right) Q+\mathcal{O}\left(\frac{1}{m_{Q}^{2}}, \frac{\vec{v}^{4}}{m_{Q}}\right) .
$$

The two-loop relation between this $m_{Q}(\mu)$ and the $\overline{\mathrm{MS}}$ mass is

$$
\begin{gathered}
m_{Q}(\mu)=\bar{m}\left\{1+\frac{4}{3} \frac{\alpha_{s}(\bar{m})}{\pi}\left(1-\frac{4}{3} \frac{\mu}{\bar{m}}-\frac{\mu^{2}}{2 \bar{m}^{2}}\right)\right. \\
\left.+\left(\frac{\alpha_{s}(\bar{m})}{\pi}\right)^{2}\left[K-\frac{8}{3}+\frac{\mu}{\bar{m}}\left(\frac{8 \beta_{0}}{9} X_{1}+\frac{8 \pi^{2}}{9}-\frac{52}{9}\right)+\frac{\mu^{2}}{\bar{m}^{2}}\left(\frac{\beta_{0}}{3} X_{2}+\frac{\pi^{2}}{3}-\frac{23}{18}\right)\right]\right\}
\end{gathered}
$$

where

$$
\begin{gathered}
K=\frac{\beta_{0}}{2}\left(\frac{\pi^{2}}{6}+\frac{71}{48}\right)+\frac{665}{144}+\frac{\pi^{2}}{18}\left(2 \ln 2-\frac{19}{2}\right)-\frac{1}{6} \zeta(3), \\
X_{1}=\ln \frac{2 \mu}{\bar{m}}-\frac{8}{3}, \quad X_{2}=\ln \frac{2 \mu}{\bar{m}}-\frac{13}{6}
\end{gathered}
$$

and $\bar{m}=\bar{m}(\bar{m})$ is the $\overline{\mathrm{MS}}$ mass normalized at the scale $\bar{m}$. We neglected small terms $\sim \mu^{3} / m_{Q}^{2}$ which can be incorporated in the same way, if necessary. We used here the $\mathcal{O}\left(\alpha_{s}^{2}\right)$ relation between the pole mass and the $\overline{\mathrm{MS}}$ mass [12].

The BLM part of the $\alpha_{s}^{3}$ terms in $m_{Q}(\mu) / \bar{m}$ in Eq. (18) is given by

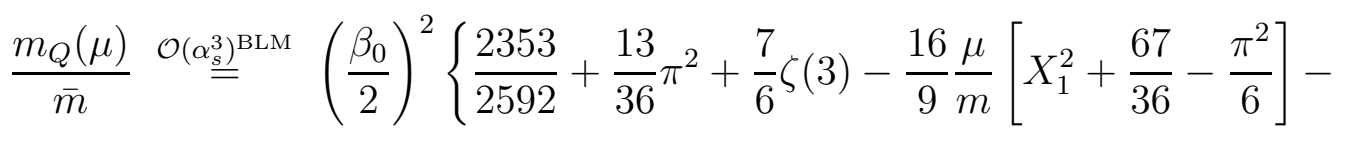




$$
\left.-\frac{2}{3} \frac{\mu^{2}}{m^{2}}\left[X_{2}^{2}+\frac{10}{9}-\frac{\pi^{2}}{6}\right]\right\}\left(\frac{\alpha_{s}}{\pi}\right)^{3},
$$

where the expressions of [0] are used.

A word of clarification is in order regarding the definition of $\alpha_{s}^{(\omega)}$. We did not specify in Eq. (4) which heavy quark masses should be used to define the reference point for $\omega$. Clearly, they must be low-scale masses, determined in one and the same way for both initial and final state quarks. The dependence on the choice then disappears in the difference $\left(m_{Q}-m_{\tilde{Q}}\right)$ in the heavy quark limit, but still persists in the kinetic energy of the recoiling quark given by the last term in (4).

Since a possible difference in the definition of $\omega$ by itself is quadratic in velocity, the choice of the normalization point for the quark masses does not matter here. This is, again, the reason why the analysis becomes so much simpler in the SV kinematics.

We conclude with addressing the question of nonperturbative effects in the dipole radiation. When the effects suppressed by powers of $\Lambda_{\mathrm{QCD}} / \omega$ are not neglected, the nonperturbative physics starts to play a role. In particular, the actual structure functions in Eqs. (2) refer to a particular heavy hadron ( $B$ meson, $\Lambda_{b}$ etc.) but not an isolated heavy quark. Likewise the complete effective coupling $\alpha_{s}^{(\omega)}$ defined in this way, as any other effective charge [14], acquires certain power-suppressed nonperturbative contribution. For example, this coupling would differ in $B$ and $\Lambda_{b}$ even in the heavy quark limit.

One can quantify the nonperturbative effects in $\alpha_{s}^{(\omega)}(\omega)$ by constructing its $1 / \omega$ expansion.] We will show now that the methods used in the OPE are applicable here.

In general, the transition amplitude $T(\omega, \vec{v})$ has a number of cuts. The physical 'decay' cut, which generates $w(\omega, \vec{v})$, starts at $\omega \simeq 0$. The other cuts go away to infinity when $m_{Q, \tilde{Q}} \rightarrow \infty$ [9]. Therefore, $\alpha_{s}^{(\omega)}(w)$ is completely determined by the analytic continuation of the nonrelativistic part of $T(\omega, \vec{v})$ to positive $\omega$. In the dispersion relation

$$
T(\omega, \vec{v})=\frac{1}{2 \pi} \int_{\omega^{\prime} \geq 0} \frac{w\left(\omega^{\prime}, \vec{v}\right)}{\omega^{\prime}-\omega-i \epsilon} d \omega^{\prime}+\frac{1}{2 \pi i} \int_{\text {other cuts }} \frac{\operatorname{disc} T\left(\omega^{\prime}, \vec{v}\right)}{\omega^{\prime}-\omega-i \epsilon} d \omega^{\prime}
$$

the contribution of the additional cuts vanishes in the heavy quark limit. At the same time, the large- $\omega$ behavior of $T(\omega)$ in the complex plane and, therefore, the asymptotics of $\alpha_{s}^{(\omega)}(\omega) / \omega$ is determined by the standard OPE expansion which expresses the contribution of the soft physics in terms of the expectation values of local heavy quark operators $\bar{Q} O_{k} Q$ of dimension $k+3$ :

$$
\frac{\delta \alpha_{s}^{(\omega)}(\omega)}{\omega}=\sum_{k} c_{k} \frac{1}{2 M_{H_{Q}}} \frac{\left\langle H_{Q}\left|\bar{Q} O_{k} Q\right| H_{Q}\right\rangle}{\omega^{k+1}} .
$$

\footnotetext{
${ }^{4}$ One should, actually, take the hadron masses in the kinematics.

${ }^{5}$ We address here only the effects of the infrared origin and discard a more theoretical problem of ultraviolet renormalons 15 or effects of small-size instantons. Thus we can merely consider, for example, the difference between the coupling from heavy mesons and heavy baryons.
} 
In this respect, the case of $\alpha_{s}^{(\omega)}$ is similar to the effective couplings defined through the Adler $D$-function $\alpha_{s}^{(D)}\left(Q^{2}\right)$ (with $D\left(Q^{2}\right) \equiv 1+\alpha_{s}^{(D)}\left(Q^{2}\right) / \pi$ ) or a similar $\alpha_{s}^{(R)}(s)$ effective coupling which measures the strong-interaction corrections in the absorptive part of the correlator of the vector currents of massless quarks in the Minkowski domain. In that cases the OPE predicts that the nonperturbative effects associated with the vacuum expectation value of the operator $G_{\alpha \beta}^{2}$, are suppressed by a fourth power of energy.

The first nontrivial heavy quark operator is the kinetic operator $\bar{Q}(i \vec{D})^{2} Q$, which, by dimensional arguments, would lead to $1 / \omega^{2}$ effects. However, it does not contribute to $\alpha_{s}^{(\omega)}$. Indeed, we can consider the initial heavy quark moving with the spacelike momentum $|\vec{p}| \ll \omega$. The structure function $w_{\vec{p}}(\omega, \vec{v})$ for such a state $\left|Q_{\vec{p}}\right\rangle$ is simply related to the one at rest, $w_{0}(\omega, \vec{v})$ :

$$
w_{\vec{p}}(\omega, \vec{v})=w_{0}(\omega-\vec{v} \vec{p}, \vec{v})+\mathcal{O}\left(1 / m_{Q}, \vec{v}^{5}\right) .
$$

If we consider a superposition of such states $\int d^{3} \vec{p} \Phi\left(\vec{p}^{2}\right)\left|Q_{\vec{p}}\right\rangle$ which is invariant under rotations, its structure function differs from $w_{0}$ only in terms $\sim \vec{p}^{2} \vec{v}^{4}$ which are negligible in the SV limit. On the contrary, the kinetic expectation value for such a state is non-zero and equals to $\left\langle\vec{p}^{2}\right\rangle$. This proves that the corresponding coefficient function $c_{2}$ must vanish.

With the heavy quark spin decoupled, only spin-singlet operators can appear in the expansion Eq. (23). There is one spin-singlet, so-called Darwin operator $O_{D}=$ $-i \bar{Q} D_{k} D_{0} D_{k} Q$ which yields $1 / \omega^{3}$-suppressed terms. [0 We do not see any reason why such terms cannot appear. Moreover, using the sum rule for the Darwin term [9, [16, 17], one finds a unique contribution associated with its logarithmic anomalous dimension:

$$
\frac{\delta_{\mathrm{D}} \alpha_{s}^{(\omega)}(\omega)}{\alpha_{s}^{(\omega)}(\omega)}=\frac{3 \pi \hat{\gamma}_{D}(\omega)}{8 \alpha_{s}^{(\omega)}(\omega)} \frac{\rho_{D}^{3}(\omega)}{\omega^{3}} \simeq\left(\frac{\alpha_{s}(\mu)}{\alpha_{s}(\omega)}\right)^{\gamma_{D} / \beta_{0}} \frac{3 \gamma_{D} \rho_{D}^{3}(\mu)}{16 \omega^{3}} \approx-\left(\frac{0.55 \mathrm{GeV}}{\omega}\right)^{3}
$$

where

$$
\begin{aligned}
& \hat{\gamma}_{D}(\mu) \rho_{D}^{3}(\mu)=\mu \frac{\mathrm{d}}{\mathrm{d} \mu} \rho_{D}^{3}(\mu), \quad \hat{\gamma}_{D}(\mu) \simeq \gamma_{D} \frac{\alpha_{s}^{(\omega)}(\mu)}{2 \pi}, \quad \gamma_{D}=-\frac{13}{2}, \\
& \rho_{D}^{3}(\mu)=\frac{\left\langle B\left|O_{D}\right| B\right\rangle_{\mu}}{2 M_{B}} \simeq \frac{2 \pi \alpha_{s}}{9} \tilde{f}_{B}^{2} M_{B} \approx 0.1 \mathrm{GeV}^{3} \text { at } \alpha_{s}(\mu) \simeq 1 .
\end{aligned}
$$

We used here the factorization estimate of $\rho_{D}^{3}$ for pseudoscalar (vector) mesons [16]; the anomalous dimensions of the $D=6$ operators were calculated in [18]. In any case, the nonperturbative effects are expected to die out quickly with energy:

$$
\delta_{\mathrm{np}} \alpha_{s}^{(\omega)}(\omega) \sim\left(\frac{\Lambda_{\mathrm{QCD}}}{\omega}\right)^{k}, \quad k \geq 3 .
$$

\footnotetext{
${ }^{6}$ At the level of nonperturbative effects, for heavy quark states with spin of light degrees of freedom $j \geq 1$ there can be the second Lorentz structure in $d(\omega) \sim(\overrightarrow{q j})^{2}$ if averaging over these spin states is not performed. Then an independent $D=6$ operator with two spacelike derivatives can appear.
} 
Even though $\alpha_{s}^{(\omega)}$ is one of many possible effective couplings in QCD, it is most appropriate for heavy flavor electroweak transitions. Moreover, it has an additional advantage compared to a few alternative ones considered in the literature, like $\alpha_{s}^{(D)}$ or $\alpha_{s}^{(R)}$. The latter determine the deviation of the corresponding observables, $D\left(Q^{2}\right)$ or $R(s)$, from their tree-level value 1 - whereas $\alpha_{s}^{(\omega)}$ directly measures the strength of the interaction. This is an obvious advantage for its accurate determination. In this respect, the two-loop relation for $\alpha_{s}^{(\omega)}$ derived in the present letter, has its counterpart in three-loop calculations of $D$-function or $R\left(\mathrm{e}^{+} \mathrm{e}^{-} \rightarrow\right.$ hadr $)$.

From this perspective, $\alpha_{s}^{(\omega)}$ is closer to the so-called $V$-scheme coupling $\alpha_{s}^{(V)}$ related to the heavy quark potential $V(R)$ [19, 4,20 . The effective coupling $\alpha_{s}^{(V)}$ is physically appropriate in quarkonia and near-threshold perturbative calculations. However, its exact definition in higher orders is not evident at the moment. As a matter of fact, it requires knowledge of the heavy quark potential at all distances $R$ including $R \rightarrow \infty$, even when $\alpha_{s}^{(V)}\left(q^{2}\right)$ is evaluated in the perturbative domain. In particular, appearance of terms $\alpha_{s}^{k} \ln \alpha_{s}$ with $k \geq 4$ in its relation to the $\alpha_{s}^{\overline{\mathrm{MS}}}[19$ may indicate that this is not a genuinely short-distance quantity. In contrast, $\alpha_{s}^{(\omega)}$ is completely defined, not only in the perturbation theory but even beyond it. This clearly represents a certain theoretical advantage.

One aspect of $\alpha_{s}^{(\omega)}$ must be noted: it is defined in Minkowski space and in this respect is similar to $\alpha_{s}^{(R)}$ but not to $\alpha_{s}^{(D)}$. Since, as was mentioned, in this problem the Euclidean OPE is still applicable, it is not too important. Nevertheless, the so-called "exponential" terms in $\alpha_{s}^{(D)}$ related to the asymptotic nature of the power expansion of the OPE, which are truly exponentially suppressed in the Euclidean domain, may yield only oscillating power-suppressed component in $\alpha_{s}^{(R)}$, and, similarly in $\alpha_{s}^{(\omega)}$. The recent theoretical discussion can be found in [21]. The coupling $\alpha_{s}^{(\omega)}$ may also show some (smeared) resonance structure at the heavy flavor thresholds.

The Euclidean counterpart of $\alpha_{s}^{(\omega)}$ can also be defined in a straightforward way. One can either use a Borel transform image of the structure function $\alpha_{s}^{(\omega)}(\omega) / \omega$, or usual dispersion integral. These technical details will be given elsewhere.

The $\overline{\mathrm{MS}}$ coupling $\alpha_{s}^{\overline{\mathrm{MS}}}$ proved to be indispensable for complicated multi-loop calculations. On the other hand, it is rather unphysical in some respects and its use as an expansion parameter sometimes obscures the perturbative expansion [22]. At the moment the complete $\mathcal{O}\left(\alpha_{s}^{2}\right)$ relation between $\alpha_{s}^{\overline{\mathrm{MS}}}$ and $\alpha_{s}^{(\omega)}$ is enough. The order$\alpha_{s}^{3}$ calculation seems feasible as well.] It is natural to think that using $\alpha_{s}^{(\omega)}$ or its Euclidean counterpart is advantageous in heavy quark decays, and may improve the accuracy of the perturbative estimates.

To summarize, we derived the non-Abelian dipole radiation by nonrelativistic color particle to second order in the strong coupling. By virtue of the SV sum rules this determines the two-loop power mixing of the number of spin-singlet effective local heavy quark operators. We give the two-loop relation between the gauge-invariant

\footnotetext{
${ }^{7}$ It is interesting to attempt calculating the perturbative corrections to $\alpha_{s}^{(\omega)}$ by considering dipole radiation in the nonlinear non-Abelian classical colordynamics.
} 
short-distance low-scale heavy quark mass $m_{Q}(\mu)$ suitable for the Wilson OPE and the value of $m_{Q}\left(m_{Q}\right)$ in the $\overline{\mathrm{MS}}$ scheme. We discuss the properties of the effective dipole radiation coupling $\alpha_{s}^{(\omega)}$ which is useful for heavy quark physics.

Acknowledgments: N.U. is grateful to I. Bigi, M. Shifman and A. Vainshtein for fruitful collaboration and to S. Brodsky, Yu. Dokshitzer, A. Mueller and M. Voloshin for illuminating discussions of perturbative aspects. This work was supported in part by NSF under the grant number PHY 92-13313, by BMBF under grant number BMBF-057KA92P and by Graduiertenkolleg "Teilchenphysik" at the University of Karlsruhe.

\section{References}

[1] M. Voloshin and M. Shifman, Yad. Fiz. 47 (1988) 801 [Sov. J. Nucl. Phys. 47 (1988) 511].

[2] M.B. Voloshin, Nucl. Phys. B154 (1979) 365;

K. Gottfried, Phys. Rev. Lett. 40 (1978) 598.

[3] A. Czarnecki and K. Melnikov, hep-ph/9706227.

[4] S.J. Brodsky, G. Lepage and P. Mackenzie, Phys. Rev. D28 (1983) 228.

[5] S. Catani and L. Trentadue, Nucl. Phys. B327 (1989) 323.

[6] N.G. Uraltsev, Nucl. Phys. B491 (1997) 303.

[7] For a detailed discussion, see P. Ball, M. Beneke and V.M. Braun, Nucl. Phys. B452 (1995) 563.

[8] I. Bigi, M. Shifman, N. Uraltsev, A. Vainshtein, Phys. Rev. D50 (1994) 2234.

[9] I. Bigi, M. Shifman, N.G. Uraltsev, hep-ph/9703290, to appear in Ann. Rev. Nucl. Part. Sci.

[10] I. Bigi, M. Shifman, N. Uraltsev and A. Vainshtein, hep-ph/9704245, to appear in Phys. Rev. D.

[11] A. Czarnecki, K. Melnikov and N. Uraltsev, hep-ph/9706311.

[12] N. Gray, D.J. Broadhurst, W. Grafe and K. Schilcher, Z. Phys. C48 (1990) 673.

[13] I. Bigi, M. Shifman, N. Uraltsev, A. Vainshtein, Phys. Rev. D52 (1995) 196.

[14] G. Grunberg, Phys.Lett. 95B (1980) 70; E - ibid. 110B (1982) 501; Phys. Rev. D29 (1984) 2315.

[15] A.I. Vainshtein and V.I. Zakharov, Phys. Rev. Lett. 73 (1994) 1207. 
[16] I. Bigi, M. Shifman, N. Uraltsev and A. Vainshtein, Int. Journ. Mod. Phys. A9 (1994) 2467.

[17] I. Bigi, A. Grozin, M. Shifman, N. Uraltsev and A. Vainshtein, Phys. Lett. B339 (1994) 160 ;

C.-K. Chow and D. Pirjol, Phys. Rev. D53 (1996) 3998.

[18] M. Voloshin and M. Shifman, ZhETF 91 (1986) 1180 [JETP 64 (1986) 698]. There is a misprint in the text preceeding Eq. (18).

[19] T. Appelquist, M. Dine and I. Muzinich, Phys. Lett. B69 (1977) 231 and Phys. Rev. D17 (1978) 2074.

[20] M. Peter, Phys. Rev. Lett. 78 (1997) 602 and hep-ph/9702245.

[21] A recent dedicated discussion is given in: B. Chibisov, R. Dikeman, M. Shifman and N. Uraltsev, Int. J. Mod. Phys. A12 (1997) 2075.

[22] S.J. Brodsky and H.J. Lu, hep-ph/9606322, hep-ph/9409462. 